\title{
Divergence by Depth in an Oceanic Fish
}

Despite the striking physical and environmental gradients associated with depth variation in the oceans, relatively little is known about their impact on population diversification, adaptation and speciation. Changes in light associated with increasing depth are likely to alter the visual environment of organisms, and adaptive changes in visual systems may be expected. The pelagic beaked redfish, Sebastes mentella, exhibits depth-associated patterns of substructure in the central North Atlantic, with a widely distributed shallow-pelagic population inhabiting waters between 250 and 550m depth and a deep-pelagic population dwelling between 550 and $800 \mathrm{~m}$. Here we performed a molecular genetic investigation of samples from fish collected from 'shallow' and 'deep' populations, using the mitochondrial control region and the gene coding for the visual-pigment rhodopsin. We identify patterns suggestive of potential adaptation to different depths, by detecting a specific amino acid replacement at the rhodopsin gene. Mitochondrial DNA results reflect a scenario of long-term demographic independence between the two $S$. mentella groups, and raise the possibility that these 'stocks' may in fact be two incipient species. 


\section{Divergence by Depth in an Oceanic Fish}

2 Peter Shum $^{1}$, Christophe Pampoulie ${ }^{2}$, Carlotta Sacchi ${ }^{3}$, and Stefano Mariani ${ }^{1^{*}}$

$3 \quad{ }^{1}$ School of Environment \& Life Sciences, University of Salford, Manchester, UK

$4 \quad{ }^{2}$ Marine Research Institute, Reykjavík, Iceland

$5 \quad{ }^{3}$ School of Biology \& Environmental Science, University College Dublin, Dublin, Ireland

$6 *$ Corresponding Author: s.mariani@salford.ac.uk

\section{Introduction}

8 Speciation phenomena in taxa diverging with gene flow, in the absence of obvious geographic 9 barriers, remain a central focus in evolutionary biology (Bird et al., 2012; Smadja \& Butlin, 2011;

10 Fitzpatrick, Fordyce \& Gavrilets, 2008). The classical model of allopatric speciation involves the

11 evolution of reproductive isolation as a result of physical barriers that block gene flow in spatially

12 separated populations; whereas populations diverging in sympatry lead to the formation of

13 species from a single panmictic population which must exhibit strong divergent selection in order

14 to overcome the homogenizing effects of gene flow (Gavrilets, 2003). Parapatric speciation

15 represents an intermediate scenario of species formation whereby partial yet restricted contact

16 zones exist between two populations with limited gene exchange (Gavrilets, 2003). Although

17 exhaustive frameworks exist to identify and interpret speciation dynamics (Rettelbach et al., 18 2013; Bird et al., 2012; Smadja \& Butlin, 2011), only recently has empirical attention been

19 directed to the role of depth gradients in aquatic (Vonlathen et al., 2009) and especially oceanic

20 biota (Roy, Hurlbut \& Ruzzante, 2012; Bird et al., 2011; Brokovich et al., 2010; Hyde et al., 21 2008). 
22 The strong physical gradients across depth layers in the ocean pose strong selective pressures in 23 aquatic organisms (Ingram, 2011; Somero, 1992), and one notable factor is the change in the light 24 environment (Warrant \& Locket, 2004), which affects vision. Visual sensitivity in marine 25 vertebrates depends on the spectral tuning mechanism of the visual pigment (VP) (Yokoyama, 26 2000), which consists of an opsin protein (part of the largest family of G-protein-coupled 27 receptors) bound to a light-sensitive chromophore. Differently-charged amino acid (AA) residues 28 in the opsin will result in slightly different light absorbance by the photoreceptor cells 29 (Yokoyama, 2002).

30 The percomorph marine family of rockfishes (Sebastidae) have played a central role in the 31 understanding of depth-associated population divergence and speciation in the ocean (Ingram, 32 2011; Stefánsson et al., 2009a; Hyde et al., 2008; Alesandrini \& Bernardi, 1999), and evidence 33 exists that the rhodopsin gene may have evolved in response to different light environments in the 34 main, ancient radiation of the genus, in the Pacific (Sivasundar \& Palumbi, 2010). North-Atlantic 35 Sebastes have a much more recent history (Hyde \& Vetter, 2007), with the four recognised extant 36 species having diversified during the Pleistocene (Bunke, Hanel, \& Trautner, 2012). In particular, 37 the beaked redfish, Sebastes mentella, consists of two genetically distinguishable groups 38 (Stefánsson et al., 2009a; Pampoulie \& Daníelsdóttir, 2008): a widely-distributed shallow-pelagic 39 (SP) form, found between 250 and 550m depth and a more circumscribed deep-pelagic (DP) 40 component, between 550 and $800 \mathrm{~m}$. However, doubts remain as to the forces at play and the time 41 scales associated with this divergence (Stefánsson et al., 2009b; Cadrin et al., 2010).

42 Here, we sought to investigate whether on-going processes of adaptation to different depth layers 43 may leave a signature of disruptive selection in the rhodopsin gene in a recently diversifying 44 Sebastes species. We also employed for the first time the mitochondrial DNA control region to 45 reconstruct historical demography and to further elucidate the evolutionary relationships between 46 'shallow' and 'deep' pelagic beaked redfish. 


\section{Material and Methods}

\section{Generation of molecular data}

49 Archive samples were randomly selected from 25 shallow-pelagic (SP) (collected above $400 \mathrm{~m}$ depth) and 25 deep50 pelagic (DP) (collected below 700m) Sebastes mentella from the Irminger Sea, south-west of Iceland previously

51 genotyped by Stéfansson et al. (2009b) (sample numbers $4 \& 5$ in the original article). DNA was isolated from gill 52 tissue that had been preserved in $96 \%$ EtOH using a modified salt extraction protocol (Miller et al., 1988) or the 53 DNeasy kit (Qiagen (C) following the manufacturer's protocol. The non-coding mitochondrial control region was 54 amplified by PCR using primers developed by Hyde \& Vetter (2007); D-RF: 5'-CCT GAA AAT AGG AAC CAA 55 ATG CCA G-3' and Thr-RF: 5'-GAG GAY AAA GCA CTT GAA TGA GC-3'. The primers by Chen et al. (2003); Rh193: 5'-CNT ATG AAT AYC CTC AGT ACT ACC-3' and Rh1039r: 5'-TGC TTG TTC ATG CAG ATG TAG A-3'

57 were used to amplify 744 bp of the intron-free rhodopsin gene in 10 shallow-pelagic $S$. mentella, 12 deep-pelagic $S$.

58 mentella, and 3 and 4 individuals of $S$. marinus and $S$. viviparus as outgroups. Reactions were carried out in $25 \mu 1$

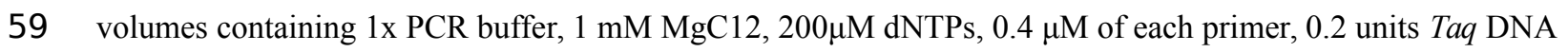

60 polymerase, with $1 \mu \mathrm{l}$ of DNA template for mtDNA (4 $\mu$ l for rhodopsin). Amplifications were performed in a

61 Biometra T3000 Thermocycler using the following temperature profiles: control region: $94^{\circ} \mathrm{C}(2 \mathrm{~min}), 35 \mathrm{cycles}$ of

$62\left[94^{\circ} \mathrm{C}(30 \mathrm{sec}), 59^{\circ} \mathrm{C}(60 \mathrm{sec}), 72^{\circ} \mathrm{C}(60 \mathrm{sec})\right]$, followed by 3 minutes at $72{ }^{\circ} \mathrm{C}$; for rhodopsin: $95^{\circ} \mathrm{C}(5 \mathrm{~min}), 37$

63 cycles of $\left[94^{\circ} \mathrm{C}(20 \mathrm{sec}), 58^{\circ} \mathrm{C}(30 \mathrm{sec}), 72^{\circ} \mathrm{C}(45 \mathrm{sec})\right]$, followed by 5 minutes at $72{ }^{\circ} \mathrm{C}$. A negative control was

64 included in all reactions. PCR products were subjected to electrophoresis through a $1 \%$ agarose gel $1 \mathrm{X}$ Tris-Borate-

65 EDTA Buffer, stained with SYBR green for visualisation via a UV-transilluminator and then purified through the

66 addition of exonuclease I and shrimp alkaline phosphatase to remove unincorporated primers and deoxynucleotides

67 in preparation to sequencing. Purified products were sequenced by Macrogen (Macrogen, Amsterdam, Europe;

68 http://dna.macrogen.com/eng/).

69 Data Analysis

70 Genetic diversity and population differentiation

71 The mtDNA control region was examined for nucleotide and haplotype diversity. This included the number of net

72 nucleotide substitutions per site between populations $\left(D_{\mathrm{a}}\right)$ which was calculated using DnaSP v5.10 (Librado \&

73 Rozas, 2009). We estimated the level of genetic variation between populations calculating pairwise population $F_{\mathrm{ST}}$ 
74 and $\Phi$ st values performed in ARLEQUIN v3.5.1.2 (Excoffier \& Lischer, 2010) with significant values tested by

755000 permutations.

76 Mismatch analysis was performed to examine the demographic history between the shallow-pelagic and deep-pelagic

77 S. mentella populations using ARLEQUIN and distributions were compared with a two-sample Kolmogorov-

78 Smirnov (K-S) test. For populations at stationary demographic equilibrium, theoretical and empirical studies show

79 that the mismatch distributions usually have multimodal, ragged or erratic distributions, and are typically smoother

80 or unimodal for populations that have undergone a recent expansion (Rogers \& Harpending, 1992). To test the

81 goodness-of-fit distribution, we calculated the sum of squared deviations (SSD) and raggedness index (r) for a

82 stepwise expansion model for the data tested by Monte Carlo Markov Chain simulations (1000 steps) in

83 ARLEQUIN.

84 Haplotype genealogies for the $S$. mentella data set were constructed following a method described by Salzburger et

85 al. (2011) based on a maximum likelihood tree for mtDNA and rhodopsin genes sequences.

86 Data from a selected suite of 12 microsatellite loci previously used for genotyping by Stéfansson et al. (2009b) were

87 used to calculate pairwise genetic differentiation (Weir \& Cockerham's $F_{\mathrm{ST}}$, Hedricks $G$ ' ${ }_{\mathrm{ST}} \&$ Jost's $D_{\text {est }}$ ) between

88 populations with 9999 permutations carried out to obtain significance levels using GenAIEx 6.501 software (Peakall

$89 \&$ Smouse, 2006). Population structure was visualized by correspondence analysis (CA) using GENETIX 4.05

90 (Belkhir et al. 1996).

91 Phylogenetic analysis and test for positive selection

92 Maximum-likelihood (ML) analyses of the rhodopsin gene sequences were performed using PhyML 3.0 (Guindon \& 93 Gascuel, 2003) under 1,000 replications; using Modeltest3.7 (Posada \& Crandall, 1998), the model that best fit the 94 data was found to be F81+I (pinvar=0.9770). Trees generated from these results were used for a test for positive 95 selection at the rhodopsin gene, conducted using the Creevey-McInerney method (Creevey \& McInerney, 2002) 96 implemented in CRANN (Creevey \& McInerney, 2003). This test is a more sensitive tree-based analysis derived

97 from the relative ratio test (McDonald \& Kreitmann, 1991). Given an appropriate rooted tree, the number of 98 synonymous and non-synonymous substitutions are calculated along each internal branch using the reconstructed 99 ancestral sequences. The method uses statistical tests for independence ( $\chi^{2}$ G-test or Fisher's exact test) to evaluate 
100 whether the ratio between synonymous (Silent Invariable (SI) to Silent Variable (SV)) and non-synonymous

101 (Replacement Invariable (RI) to Replacement Variable (RV)) substitutions deviate from the expected value under the

102 neutral model. Where the G-test fails to produce a result, the Fisher's test is used and vice versa. Positive directional

103 selection is expected if there is a significantly higher number of RI substitutions or non-directional selection if

104 RV>RI. The test was performed using S. alutus as outgroup (Fig. 3) as it represents the closest common ancestor for 105 the Atlantic Sebastes spp. (Hyde \& Vetter, 2007).

\section{Results and Discussion}

107 We recovered 16 mtDNA haplotypes, defined by 15 total variable sites across the two groups.

108 Haplotypes were almost completely segregated between depth layers, with eight haplotypes 109 found in the deep, never recovered in the shallow area, and resembling a starburst pattern, three

110 mutational steps away from the rest of the network (Fig. 1a). Of the 10 haplotypes found in the

111 shallow, eight were exclusive of this habitat, and only two individuals collected in the deep were

112 found to bear a 'shallow-type' sequence. The shallow-pelagic (SP) group exhibited much greater

113 diversity $(\hat{\mathrm{h}}=0.887 \pm 0.033, \pi=0.00504 \pm 0.00082)$ than the deep-pelagic (DP) group

$114(\hat{\mathrm{h}}=0.543 \pm 0.119, \pi=0.00238 \pm 0.00071)($ Table 1$)$.

115 Partitioning of genetic variance between the populations showed highly significant and strong

116 population structure (Table. 2). The mismatch distributions of the two groups (Fig. 1b) were 117 significantly different (K-S test: $D_{300}=0.3, \mathrm{p}<<0.001$ ), and confirmed what is visually apparent

118 from the haplotype network: a scenario of more recent and pronounced demographic and spatial

119 expansion in the deep-pelagic group compared to the shallow-pelagic (Table 3). Using Nei's (Nei,

120 1987) formula for divergence time: $T=D_{\mathrm{a}} / 2 \mu$, where $2 \mu$ represents a general mtDNA evolutionary

121 rate, commonly assumed to be around 11\% per million years for fish mtDNA control region 
122 (Patarnello, Volckaert \& Castilho, 2007), we find that the "deep" and "shallow" lineages split

123 over 44,000 years ago.

124 The rhodopsin gene was tested for signatures of positive selection using the Creevey-McInerney 125 method rooting the tree with $S$. alutus as an outgroup (Fig. 2). Values for the four substitution 126 variables, G-test, and $p$-values along each branch are presented in Table 4. Two branches 127 (numbers 26 and 27) showed significance at the $p=0.05$ level (Fig. 2). Branches 26 and 27 show 128 significant RI to RV deviations from neutrality, due to non-synonymous substitutions (Table 3), 129 suggesting that positive disruptive selection is acting on the rhodopsin gene for the clade and on 130 the internal branch leading to the shallow group. We observed a fixed non-synonymous AA 131 substitution within the transmembrane domain, which strongly discriminates the two groups 132 inhabiting shallow and deep environments (Fig. 3). The shallow-pelagic group exhibits a GTC at 133 position 119, which codes for Valine (L119V), while the deep-pelagic type displays an ATC, 134 coding for Isoleucine (L119I). Amino acid changes located in the transmembrane helical regions 135 have been known to be important for spectral tuning (Yokoyama et al., 2008), yet in-vitro 136 experimental spectral analyses of vertebrate rhodopsins suggest that amino acid substitutions at 137 site 119 have negligible effect on absorption spectra (Yokoyama et al., 2008). Nevertheless, 138 substitutions within the transmembrane protein domain III (helix-III), such as site 119, have been 139 shown to affect the decay rate of metarhodopsin II ("meta II"; Ou et al., 2011), which is an 140 intermediate of rhodopsin that binds and activates transducin, the visual G-protein (Smith, 2010).

$141 \mathrm{Ou}$ et al. (2011) have shown that an AA replacement L119C against the wild-type rhodopsin 142 resulted in shorter meta II lifetimes, suggesting more responsive structural alterations at the helix' 143 G-protein binding site.

144 Neither spectral nor conformational analyses have so far been conducted on shallow-pelagic and 145 deep-pelagic Sebastes mentella, but the AA variation observed here could underlie differential 
146 hydrophobic activity and photoisomerization sensitivity (Ou et al., 2011) that could hold some

147 adaptive value, even without net change in wavelength of maximal absorption.

148 Sivasundar and Palumbi (2010) discovered a striking association between AA replacements along 149 the rhodopsin gene and inferred depth preference in many North Pacific Sebastes. Interestingly, 150 four North-Pacific Sebastes (S. chlorostictus, S. elongates, S. aurora and S. melanostomus) 151 typically associated with deeper waters were observed to exhibit the same AA replacement L119I 152 as detected in the deep-sea $S$. mentella. Similarly, one Pacific species (S. diploproa) exhibits the 153 AA replacement L119V, which is linked to a shift back to shallower waters, and mirrors the 154 polymorphism in the shallow-pelagic $S$. mentella.

155 Although larger sample sizes will be required in the future to test these patterns more robustly, 156 the implications of these findings are twofold, and have powerful resonance for both marine 157 evolution and fisheries management. First, mitochondrial variation between 'shallow' and 'deep' $158 S$. mentella in the North Atlantic unveil a degree of historical divergence that previously 159 employed genetic markers either failed to detect (Bunke et al., 2012) or could not reliably frame 160 in a phylogeographic context (Stefánsson et al., 2009b).

161 The level of differentiation and haplotype sorting is such that evolutionary independence can be 162 broadly upheld for these two habitat-segregated lineages, and re-analysis of microsatellite data 163 confirm this picture (Fig. 4). In particular, the comparison of frequency-based indicators of 164 substructure $\left(F_{\mathrm{ST}}\right.$ for mtDNA, and $G^{\prime}{ }_{\mathrm{ST}}$ and $D_{\text {est }}$ to account for the hypervariability of 165 microsatellite loci) reveal values (Table 2) that match theoretical expectations under neutral 166 divergence, taking into account the four-fold strength of genetic drift at mitochondrial markers. 167 Interestingly, we also noticed two individuals with a 'shallow-type' mtDNA haplotype, which 168 were caught in the deep layer. One of these two (DP29, Fig. 2) also screened at the rhodopsin 169 locus, exhibits a sequence typical of the shallow layer, and its multilocus microsatellite genotype 
170 also falls with the shallow group (Fig. 4), which can be interpreted as the occurrence of

171 individual movements along the water column during the life cycle (i.e. short-term "dives" into

172 the deep, by shallow-dwelling fish). Another deep-caught individual (DP1) also exhibits a

173 "shallow" haplotype, but a "deep-like" ATC rhodopsin sequence and an inconclusive multilocus

174 microsatellite genotype (Fig. 4). Collectively, this likely reflects the occurrence of introgressive

175 hybridisation between the two groups, as previously suggested by Pampoulie \& Danielsdottir 176 (2008).

177 Furthermore, the stark pattern of depth-associated divergence at the rhodopsin gene is perhaps 178 even more surprising, were it not for the fact that comparable evolutionary genetic patterns have 179 recently been credited with a key role in the diversification of the more ancient Pacific Sebastes 180 group (Sivasundar \& Palumbi, 2010). It has been hypothesized that fast-evolving markers will 181 allow to determine recent speciation events for closely related Sebastes spp. (Alesandrini \& 182 Bernardi, 1999; Cadrin et al., 2010). The present data provide a snapshot of the evolutionary 183 mechanisms that may be at play in the young, species-poor, Atlantic Sebastes lineage, during its 184 initial phase of adaptive radiation, underpinned by positive selection at the rhodopsin gene.

185 Less than a decade ago, S. mentella was assumed to be panmictic in the North Atlantic, and the 186 rapidly increasing fishery pressure on these stocks did not recognise any possible substructure 187 until 2009 (Cadrin et al., 2010). These latest results dismiss the notion of panmixia in this oceanic 188 species, and, perhaps more intriguingly, open the possibility that the two 'shallow' and 'deep' 189 groups may represent two lineages experiencing adaptation towards divergent environmental 190 conditions. In the near future, it should be experimentally evaluated whether the amino acid 191 replacements at the 119 position actually produce detectable changes in retinal absorbance or 192 structural responsiveness, and whether more powerful molecular comparisons covering a wider 
193 portion of the genome (e.g. SNP-based genome scans; transcriptomic approaches) will offer 194 further insights into the role of depth as a diversifying agent in the ocean.

\section{Acknowledgements}

196 We are grateful to the WKREDS workshop of the International Council for the Exploration of the 197 Sea (ICES) for inspiring this work. We are also indebted to Valerie Chosson for technical 198 assistance at the MRI, and Emma Teeling, Bruno Fonseca Simões and three reviewers, for the 199 constructive criticism offered. 
200

201

202

203

204

205

206

207

208

209

210

211

212

213

214

215

216

217

218

219

220

221

222

223

224

225

\section{References}

Alesandrini S, Bernardi G. 1999. Ancient species flocks and recent speciation events: what can rockfish teach us about cichlids (and vice versa)? Journal of Molecular Evolution 49:814818.

Belkhir K, Borsa P, Chikhi L, Raufaste N, \& Bonhomme F. 1996. GENETIX 4.05, logiciel sous Windows TM pour la génétique des populations. Laboratoire Génome, Populations, Interactions, CNRS UMR 5000, Université de Montpellier II, Montpellier (France).

Bird CE, Fernandez-Silva I, Skillings DJ, Toonen RJ. (2012) Sympatric speciation in the post "modern synthesis" era of evolutionary biology. Evolutionary Biology 39: 158-180.

Bird CE, Holland BS, Bowen BW, Toonen RJ. 2011. Diversification of sympatric broadcastspawning limpets (Cellana spp.) within the Hawaiian archipelago. Molecular Ecology 20: 2128-2141.

Brokovich E, Ben-Ari T, Kark S, Kiflawi M, Dishon G, Iluz D, Shashar N. 2010. Functional changes of the visual system of the damselfish Dascyllus marginatus along its bathymetric range. Physiology and Behavior 101: 413-421.

Bunke C, Hanel R, Trautner JH. 2012. Phylogenetic relationships among North Atlantic redfish (genus Sebastes) as revealed by mitochondrial DNA sequence analysis. Journal of Applied Ichthyology 29:82-92.

Cadrin SX, Bernreuther M, Daníelsdóttir AK, Hjörleifsson E, Johansen T, Kerr L, Kristinsson K, Mariani S, Nedreaas K, Pampoulie C, Planque B, Reinert J, Saborido-Rey F, Sigurðsson T, Stransky C. 2010. A Population structure of beaked redfish, Sebastes mentella: evidence of divergence associated with different habitats. ICES Journal of Marine Science 67:16171630.

Chen W-J, Bonillo C, Lecointre G. 2003. Repeatability of clades as a criterion of reliability: a case study for molecular phylogeny of Acanthomorpha (Teleostei) with larger number of taxa. Molecular Phylogenetics and Evolution 26: 262-288. 
226 Creevey CJ, McInerney JO. 2002. An algorithm for detecting directional and non-directional 227 positive selection, neutrality and negative selection in protein coding DNA sequences. Gene $228 \quad 300: 43-51$.

229 Creevey CJ, McInerney JO. 2003. CRANN: detecting adaptive evolution in. Bioinformatics $230 \quad 19: 1726$.

231 Excoffier L, Lischer HE. 2010. Arlequin suite ver 3.5: a new series of programs to perform 232 population genetics analyses under Linux and Windows. Molecular Ecology Resources 10: $233 \quad 564-567$.

234 Fitzpatrick BM, Fordyce JA, Gavrilets S. 2008. What, if anything, is sympatric speciation? 235 Journal of Evolutionary Biology 21:1452-1459.

236 Gavrilets S. 2003. Perspective: models of speciation: what have we learned in 40 years? 237 Evolution 57:2197-2215.

238 Guindon S, Dufayard JF, Anisimova M., Hordijk W, Gascuel O. 2010. PhyML 3.0: new algoriths, 239 methods and utilities. Systematic Biology 59:307-321.

240 Hyde JR, Kimbrell CA, Budrick JE, Lynn EA, Vetter RD. 2008. Cryptic speciation in the 241 vermilion rockfish (Sebastes miniatus) and the role of bathymetry in the speciation process. $242 \quad$ Molecular Ecology 17:1122-1136.

243 Hyde J, Vetter RD. 2007. The origin, evolution, and diversification of rockfishes of the genus 244 Sebastes (Cuvier). Molecular phylogenetics and evolution 44:790-811.

245 Ingram T. 2011. Speciation along a depth gradient in a marine adaptive radiation. Proceedings of 246 the Royal Society of London Series B 278: 613-618. 
247 Librado P, Rozas J. 2009. DnaSP v5: a software for comprehensive analysis of DNA 248 polymorphism data. Bioinformatics 25: 1451-1452.

249 McDonald JH, \& Kreitman M. 1991. Adaptive protein evolution at the Adh locus in Drosophila. $250 \quad$ Nature 351: 652-654.

251 Miller SA, Dykes DD, \& Polesky H F. 1988. A simple salting out procedure for extracting DNA 252 from human nucleated cells. Nucleic Acids Research 16: 1215.

253 Nei M. 1987. Molecular Evolutionary Genetics. Columbia University Press, New York, NY.

254 Ou WB, Yi T, Kim JM, Khorana HG. 2011. The roles of transmembrane domain Helix-III during 255 rhodopsin photoactivation. PLoS One 6:e17398.

256 Pampoulie C. Daníelsdóttir AK. 2008. Resolving species identification problems in the genus 257 Sebastes using nuclear genetic markers. Fisheries Research 93:54-63.

258 Patarnello T, Volckaert F. Castilho R. 2007. Pillars of hercules: Is the atlantic-mediterranean 259 transition a phylogeographical break? Molecular Ecology 16:4426-4444.

260 Peakall R, Smouse PE. 2006. Genalex 6: genetic analysis in Excel. Population genetic software 261 for teaching and research. Molecular Ecology Notes 6: 288-295.

262 Posada D, Crandall KA. 1998. MODELTEST: testing the model of DNA substitution. 263 Bioinformatics 14:817-818.

264 Rettelbach A, Kopp M, Dieckmann U, \& Hermisson J. 2013. Three Modes of Adaptive 265 Speciation in Spatially Structured Populations. The American Naturalist 182: E215-E234.

266 Rogers AR, \& Harpending H. 1992. Population growth makes waves in the distribution of 267 pairwise genetic differences. Molecular Biology and Evolution 9: 552-569. 
268 Roy D, Hurlbut TR, Ruzzante DE. 2012. Biocomplexity in a demersal exploited fish, white hake 269 (Urophycis tenuis): depth-related structure and inadequacy of current management 270 approaches. Canadian Journal of Fisheries and Aquatic Sciences 69:415-429.

271 Salzburger W, Ewing GB, von Haeseler A. 2011. The performance of phylogenetic algorithms in 272 estimating haplotype genealogies with migration. Molecular Ecology 20:1952-1963.

273 Sivasundar A, Palumbi SR. 2010. Parallel amino acid replacements in the rhodopsins of the 274 rockfishes (Sebastes spp.) associated with shifts in habitat depth. Journal of Evolutionary 275 Biology 23:1159-1169.

276 Smadja CM, \& Butlin RK. 2011. A framework for comparing processes of speciation in the 277 presence of gene flow. Molecular Ecology 20:5123-5140.

278 Smith SO. 2010. Structure and activation of the visual pigment rhodopsin. Annual Review of $279 \quad$ Biophysics 39:309-328.

280 Somero G N. 1992. Adaptations to high hydrostatic pressure, Annual review of physiology $281 \quad 54: 557-577$.

282 Stefánsson MO, Reinert J, Sigurðsson P, Kristinsson K, Nedreaas K, Pampoulie C. 2009a. Depth 283 as a potential driver of genetic structure of Sebastes. ICES Journal of Marine Science $284 \quad 66: 680-690$.

285 Stefánsson MÖ, Sigurðsson T, Pampoulie C, Daníelsdóttir AK, Thorgilsson B, Ragnarsdóttir R, 286 Gíslason D, Coughlan J, Cross TF, Bernatchez L. 2009b. Pleistocene genetic legacy suggests 287 incipient species of Sebastes mentella in the Irminger Sea. Heredity 102:514-524. 
288 Vonlanthen P, Roy D, Hudson AG, Largiader CR, Bittner D, Seehausen O. 2009. Divergence 289 along a steep ecological gradient in lake whitefish (Coregonus sp.). Journal of Evolutionary $290 \quad$ Biology 22:498-514.

291 Warrant EJ, Locket N. 2004. Vision in the deep sea. Biological Reviews 79:671-712.

292 Yokoyama S. 2000. Molecular evolution of vertebrate visual pigments. Progress in Retinal and 293 Eye Researh 19:385-419.

294 Yokoyama S. 2002. Molecular evolution of color vision in vertebrates. Gene 300:69-78.

295 Yokoyama S, Tada T, Zhang H, Britt L. 2008. Elucidation of phenotypic adaptations: Molecular 296 analyses of dim-light vision proteins in vertebrates. Proceedings of the National Academy of 297 science of the United State of America 105:13480-13485. 
298 Table 1. Summary of mtDNA control region molecular

299 diversity: $\mathrm{H}=$ unique haplotypes; $\mathrm{n}=$ number of individuals; $\mathrm{S}$

\begin{tabular}{ccccccccc}
\hline Population & $\mathbf{H} / \mathbf{n}$ & $\mathbf{S}$ & $\hat{\mathbf{h}} \pm \mathbf{S D}$ & $\boldsymbol{\pi} \pm \mathbf{S D}$ & $\boldsymbol{D}_{\mathbf{T}}$ & $\boldsymbol{F}_{\mathbf{S}}$ & $\boldsymbol{F}^{*}$ & $\boldsymbol{D}^{*}$ \\
\hline $\mathbf{( S P )}$ & $8 / 25$ & 10 & $0.887 \pm 0.033$ & $0.00504 \pm 0.00082$ & -1.08590 & -3.806 & -2.30037 & -2.35314 \\
$(\mathbf{D P})$ & $6 / 25$ & 8 & $0.543 \pm 0.119$ & $0.00238 \pm 0.00071$ & -1.77639 & -4.717 & -1.49744 & -1.08199
\end{tabular}

$300=$ Segregating sites; $\hat{\mathrm{h}}=$ haplotype diversity; $\pi=$ nucleotide

301 diversity (both with associated standard deviations, $\mathrm{SD}$ ); $D_{\mathrm{T}}=$

302 Tajima"s $D ; F_{\mathrm{S}}=$ Fu's $F_{\mathrm{S}}$ statistic; $F^{*}=\mathrm{Fu}$ and Li's $F$ test; $D^{*}=$

303 Fu and Li's $D$ test. $\mathrm{SP}=$ Shallow Pelagic; DP = Deep Pelagic.

304 Table 2. Analysis of fixation/differentiation indices for mtDNA and microsatellite data between 305 shallow-pelagic (SP) and deep-pelagic (DP) S mentella.

\begin{tabular}{lcccc}
\hline Marker & Group & $\begin{array}{c}\text { Fixation/Differentiatio } \\
\text { n index }\end{array}$ & $\begin{array}{c}\text { estimat } \\
\mathbf{e}\end{array}$ & $\boldsymbol{p}$ \\
\hline mtDNA & SP vs DP & $F_{\text {ST }}$ & 0.636 & $<0.001$ \\
Microsatellites & SP vs DP & $\Phi_{\text {ST }}$ & 0.273 & $<0.001$ \\
& & $F_{\text {ST }}$ & 0.031 & 0.001 \\
& & $G^{\prime}{ }_{\text {ST }}$ & 0.135 & 0.001 \\
& & $D_{\text {est }}$ & 0.121 & 0.001 \\
\hline
\end{tabular}


306 Table 3. Mismatch distribution parameter estimates for mtDNA control region

\begin{tabular}{|c|c|c|c|c|c|c|}
\hline \multirow[t]{2}{*}{ Population } & \multicolumn{5}{|c|}{ Mismatch Distribution } & \\
\hline & $\tau$ & $\theta 0$ & $\theta 1$ & SSD $p$-value \pm SD & r $p$-value \pm SD & \\
\hline (SP) & 1.9 & 0.0000 & 99,999 & $\begin{array}{l}\text { DE } 0.007 \pm 0.18 \\
\text { SE } 0.007 \pm 0.002\end{array}$ & $\begin{array}{c}0.30 \pm 0.21 \\
0.27 \pm 0.28\end{array}$ & \\
\hline (DP) & 0.1 & 0.0000 & 99,999 & $\begin{array}{l}\text { DE } 0.274 \pm 0.18 \\
\text { SE } 0.004 \pm 0.002\end{array}$ & $\begin{array}{l}0.00 \pm 0.21 \\
0.67 \pm 0.28\end{array}$ & $\tau=\operatorname{tau} ; \theta_{0}=$ theta 0 \\
\hline $\begin{array}{l}\theta_{1}=\text { theta } 1 ; \mathrm{Ss} \\
\text { statistic; } \mathrm{DE} \\
\text { pelagic. }\end{array}$ & & ed deviat & $\mathrm{s} ; \mathrm{r}=\mathrm{rag}$ & $\begin{array}{l}\text { Iness } \\
\text { tial expansion; (S }\end{array}$ & $=$ Shallow $p$ & $(D P)=$ Deep- \\
\hline
\end{tabular}


311 Table 4. Creevey-McInerney positive selection analysis on Sebastes rhodopsin sequences

312 outgrouped with S. alutus (GenBank: EF212407.1), G-value $\mathrm{p}<0.05$ for Fisher's ${ }^{\dagger}$ and G-Test*

\begin{tabular}{|c|c|c|c|c|c|}
\hline Branch no. & $\mathbf{R I}$ & $\mathbf{R V}$ & SI & SV & G-value \\
\hline \multicolumn{6}{|l|}{ rhodopsin } \\
\hline 0 & 0 & 2 & 0 & 0 & 0.00 \\
\hline 1 & 0 & 2 & 0 & 0 & 0.00 \\
\hline 2 & 0 & 2 & 0 & 0 & 0.00 \\
\hline 3 & 0 & 5 & 0 & 0 & 0.00 \\
\hline 4 & 0 & 5 & 0 & 0 & 0.00 \\
\hline 5 & 0 & 5 & 0 & 0 & 0.00 \\
\hline 6 & 0 & 5 & 0 & 0 & 0.00 \\
\hline 7 & 0 & 5 & 0 & 0 & 0.00 \\
\hline 8 & 0 & 5 & 0 & 0 & 0.00 \\
\hline 9 & 0 & 5 & 0 & 0 & 0.00 \\
\hline 10 & 0 & 5 & 0 & 0 & 0.00 \\
\hline 11 & 0 & 8 & 0 & 0 & 0.00 \\
\hline 12 & 0 & 8 & 0 & 0 & 0.00 \\
\hline 13 & 0 & 8 & 0 & 0 & 0.00 \\
\hline 14 & 0 & 8 & 0 & 0 & 0.00 \\
\hline 15 & 0 & 8 & 0 & 0 & 0.00 \\
\hline 16 & 0 & 11 & 1 & 0 & 2.01 \\
\hline 17 & 0 & 0 & 1 & 0 & 0.00 \\
\hline 18 & 0 & 9 & 0 & 0 & 0.00 \\
\hline 19 & 0 & 11 & 0 & 0 & 0.00 \\
\hline 20 & 0 & 12 & 0 & 0 & 0.00 \\
\hline 21 & 0 & 12 & 0 & 0 & 2.2 \\
\hline 22 & 0 & 12 & 1 & 0 & 2.2 \\
\hline 23 & 0 & 12 & 1 & 0 & 2.2 \\
\hline 24 & 0 & 12 & 1 & 0 & 2.2 \\
\hline 25 & 0 & 12 & 1 & 0 & 2.2 \\
\hline 26 & 1 & 12 & 2 & 0 & $5.5^{\dagger}$ \\
\hline 27 & 8 & 24 & 5 & 1 & $6.76^{*}$ \\
\hline
\end{tabular}




\section{FIGURE CAPTIONS}

314 Figure 1. Sebastes mentella genealogies for mtDNA (n=50;25 SP $+25 \mathrm{DP})$ and rhodopsin 315 ( $=22 ; 10$ SP + 12 DP), and mtDNA mismatch distributions. (A) Haplotype network for the 316 shallow (red) and deep (blue) groups for mtDNA (i) and rhodopsin (ii). The size of each circle 317 represents the proportion of haplotypes. The lengths of the connecting lines reflect the number of

318 mutations between haplotypes. (B) Mismatch distributions from the mtDNA sequences of 319 shallow (i) and deep (ii) groups, respectively from above and below 550 metres depth 320 respectively. Dotted lines (Up/low bound.) represent the $95 \%$ boundaries of the simulated 321 distributions.

322 Figure 2. Creevey-McInerney analysis of Sebastes rhodopsin. Rhodopsin reveals significant 323 positive selection $(*)$ at two nodes $(26,27)$.

324 Figure 3. Chromatograms illustrating the non-synonymous $\mathrm{A} / \mathrm{G}$ mutation on the rhodopsin gene, 325 which discriminates between "Deep-Pelagic" (a: above) and "Shallow-Pelagic" (b: below) 326 Sebastes mentella.

327 Figure 4. Correspondence analysis based on microsatellite data. Each circle represent an 328 individual, red and blue refer to the shallow-pelagic (SP) and deep-pelagic (DP) groups 329 respectively. 


\section{Figure 1}

Comprehensive image of mtDNA and Rhodopsin genetic divergence and Mismatch Distributions

Figure 1. S. mentella genealogies for mtDNA $(n=50 ; 25 S P+25$ DP) and rhodopsin $(n=22$; 10 SP + 12 DP), and mtDNA mismatch distributions. (A) Haplotype network for the shallow (red) and deep (blue) groups for mtDNA (i) and rhodopsin (ii). The size of each circle represents the proportion of haplotypes. The lengths of the connecting lines reflect the number of mutations between haplotypes. (B) Mismatch distributions from the mtDNA sequences of shallow (i) and deep (ii) groups, respectively from above and below 550 metres depth respectively. Dotted lines (Up/low bound.) represent the 95\% boundaries of the simulated distributions. 

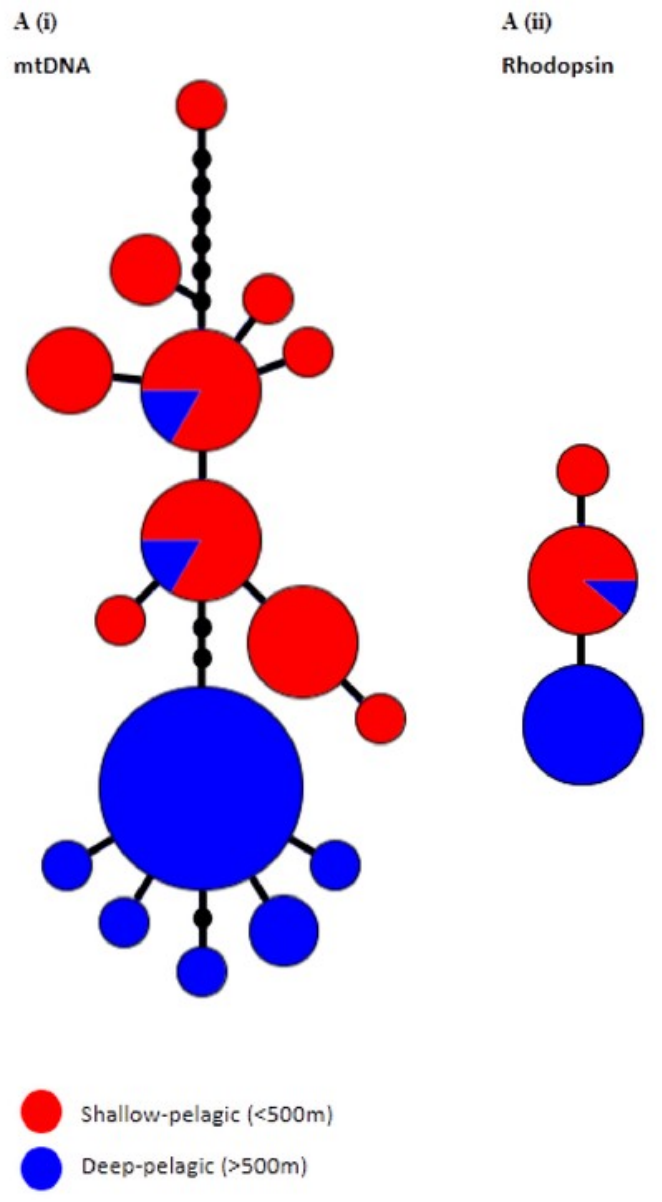

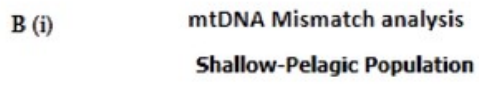
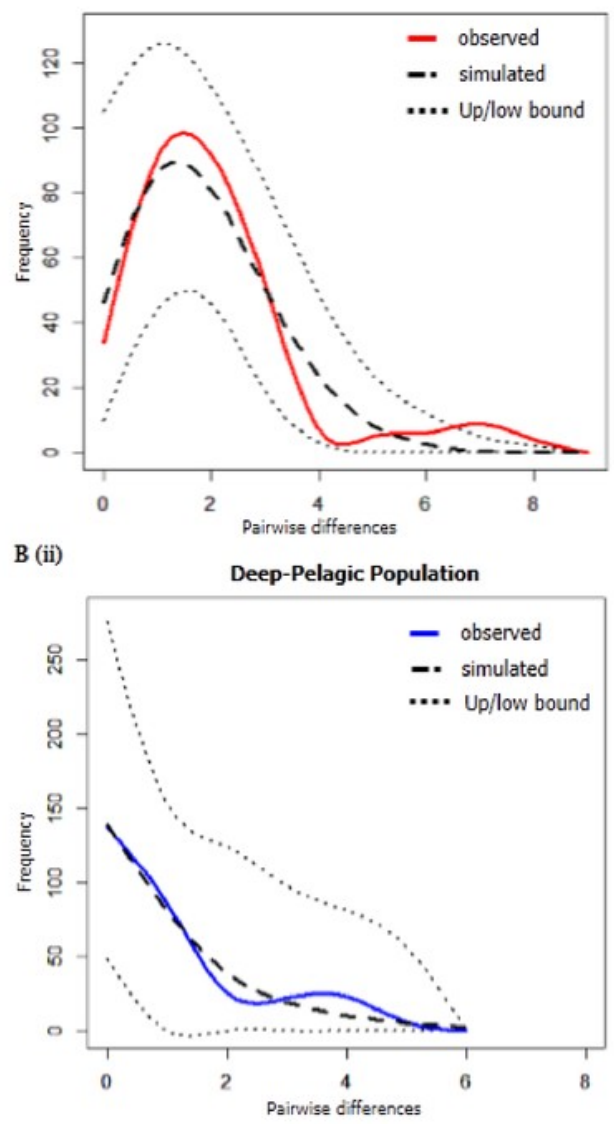


\section{Figure 2}

CRANN test tree

Creevey-Mclnerney analysis of Sebastes rhodopsin. Rhodopsin reveals significant positive selection $\left(^{*}\right)$ at two nodes $(26,27)$.

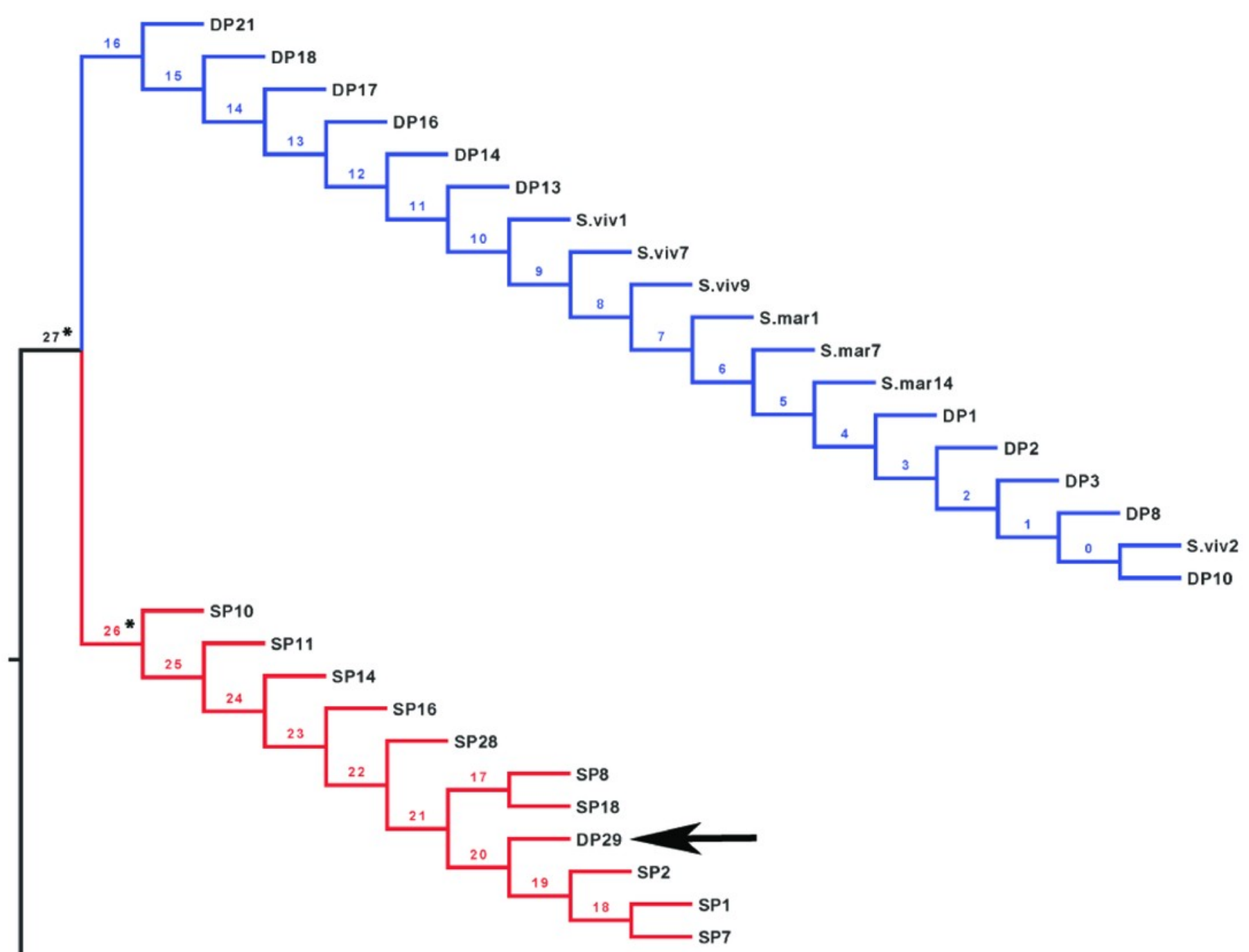

S. alutus

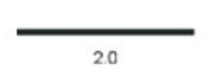




\section{Figure 3}

Example of non-synonymous base substitution

Figure 3. Chromatograms illustrating the non-synonymous $A / G$ mutation on the rhodopsin gene, which discriminates between "Deep-Pelagic" (a: above) and "Shallow-Pelagic" (b: below) Sebastes mentella.

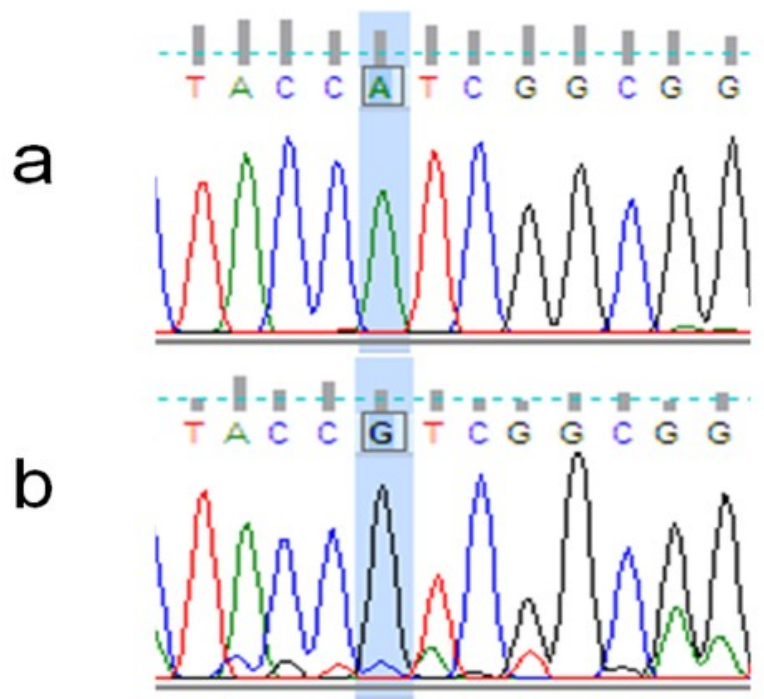




\section{Figure 4}

Ordination of microsatellite genotypes

Figure 4. Correspondence analysis based on microsatellite data. Each circle represent an individual, red and blue refer to the shallow-pelagic (SP) and deep-pelagic (DP) groups respectively.

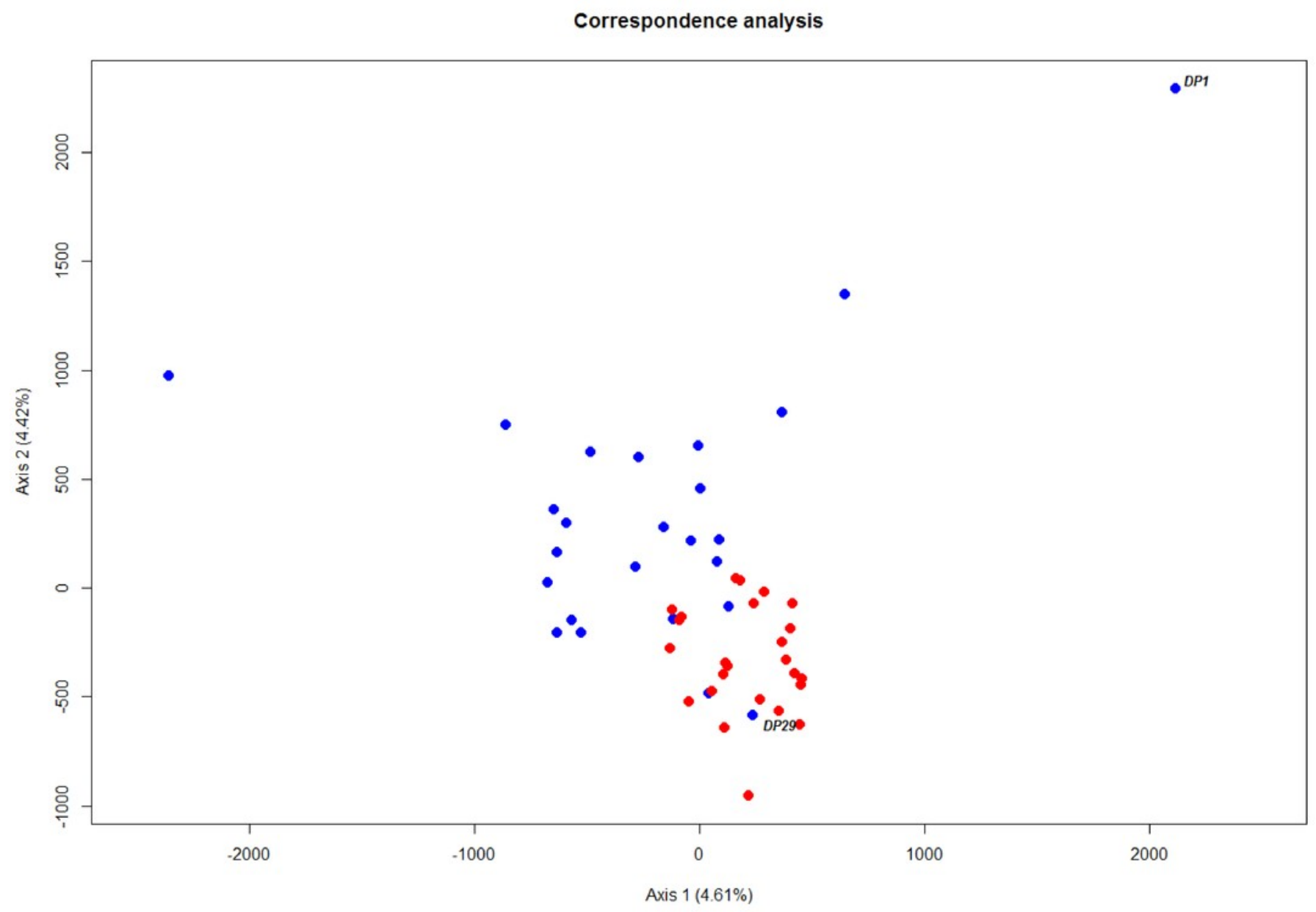




\section{Table 1 (on next page)}

Summary of mtDNA control region molecular 
Table 1. Summary of mtDNA control region molecular diversity: $\mathrm{H}=$ unique haplotypes; $\mathrm{n}=$ number of individuals; $\mathrm{S}=\mathrm{Segregating}$ sites; $\mathrm{FS}=$ Fu's FS statistic; DT = Tajima"s D ; F* = Fu and Li's F test; D* = Fu and Li's D test. SP = Shallow Pelagic; DP = Deep Pelagic.

\begin{tabular}{|c|c|c|c|c|c|c|c|c|}
\hline Population & $\mathbf{H} / \mathbf{n}$ & $\mathbf{S}$ & $\hat{\mathbf{h}} \pm \mathrm{SD}$ & $? \pm \mathrm{SD}$ & $\mathbf{D}_{\mathrm{T}}$ & $\overline{F_{S}}$ & $F^{*}$ & $D^{*}$ \\
\hline (SP) & $8 / 25$ & 10 & $0.887 \pm 0.03$ & $\begin{array}{l}0.00504 \pm 0.0008 \\
2\end{array}$ & -1.08590 & -3.806 & -2.30037 & -2.35314 \\
\hline (DP) & $6 / 25$ & 8 & $\begin{array}{l}\frac{7}{\sigma} 0.543 \pm 0.11 \\
\sum_{0} 9\end{array}$ & $\begin{array}{l}0.00238 \pm 0.0007 \\
1\end{array}$ & -1.77639 & -4.717 & -1.49744 & -1.08199 \\
\hline & & & 1) & & & & & \\
\hline
\end{tabular}




\section{Table 2 (on next page)}

Analysis of fixation/differentiation indices for mtDNA and microsatellite data between shallow-pelagic (SP) and deep-pelagic (DP) S mentella. 
Table 2. Analysis of fixation/differentiation indices for mtDNA and microsatellite data between shallow-pelagic (SP) and deep-pelagic (DP) S. mentella.

\begin{tabular}{|c|c|c|c|c|}
\hline Marker & Group & $\begin{array}{c}\text { Fixation/Differentiation } \\
\text { index }\end{array}$ & $\begin{array}{c}\text { estimat } \\
\mathrm{e}\end{array}$ & $p$ \\
\hline \multirow[t]{2}{*}{ mtDNA } & SP vs DP & $\mathrm{F}_{\mathrm{ST}}$ & 0.636 & $<0.001$ \\
\hline & & $\Phi_{\mathrm{ST}}$ & 0.273 & $<0.001$ \\
\hline \multirow[t]{3}{*}{ Microsatellites } & SP vs DP & $\mathrm{F}_{\mathrm{ST}}$ & 0.031 & 0.001 \\
\hline & & $\mathrm{G}_{\text {ST }}^{\prime}$ & 0.135 & 0.001 \\
\hline & & $D_{\text {est }}$ & 0.121 & 0.001 \\
\hline
\end{tabular}




\section{Table 3 (on next page)}

Mismatch distribution parameter estimates for mtDNA control region 
Table 3. Mismatch distribution parameter estimates for mtDNA control region

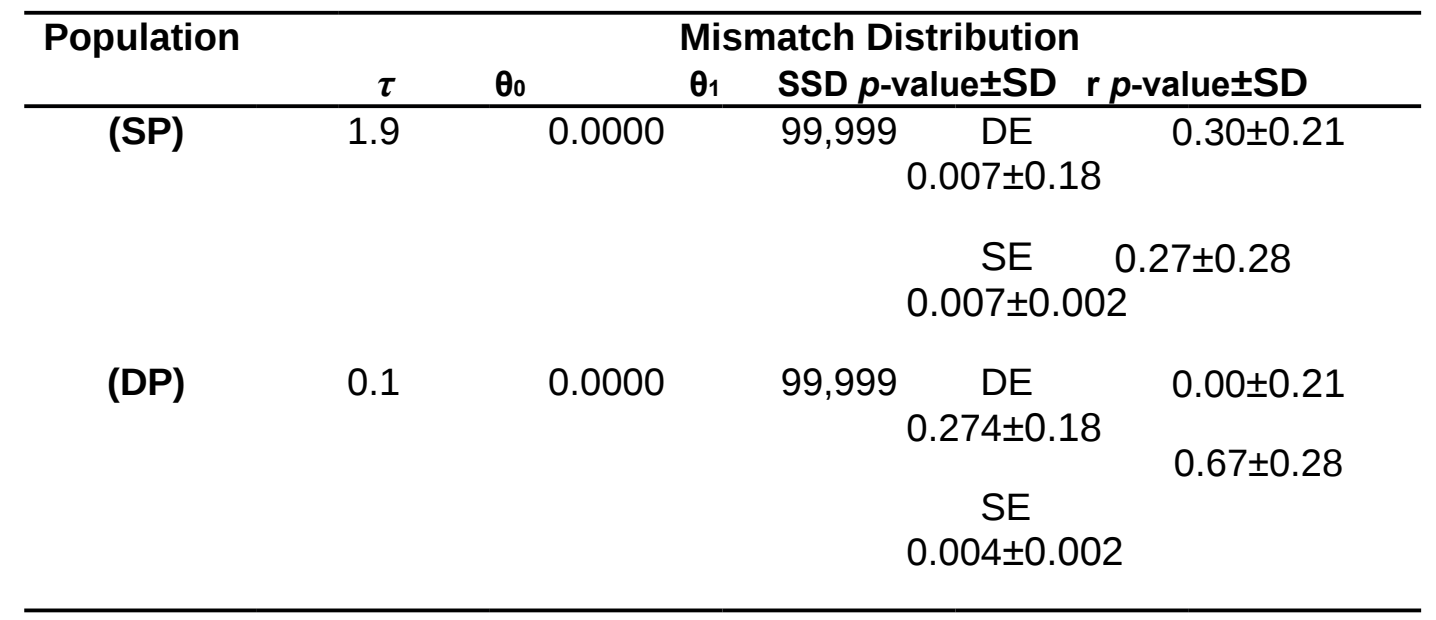

$\tau=$ tau; $\theta_{0}=$ theta $0 ; \theta_{1}=$ theta $1 ; \mathrm{SSD}=$ sum of squared deviations; $\mathrm{r}=$ raggedness

statistic; DE: demographic expansion, SE: spatial expansion; (SP) = Shallow pelagic; (DP) = Deep-pelagic. 


\section{Table 4 (on next page)}

Creevey-Mclnerney positive selection analysis on Sebastes rhodopsin sequences outgrouped with S. alutus (GenBank: EF212407.1), G-value $p<0.05$ for Fisher's $^{\ddagger}$ and GTest $^{*}$ 
Table 4. Creevey-McInerney positive selection analysis on Sebastes rhodopsin sequences outgrouped with S. alutus (GenBank: EF212407.1), G-value $p<0.05$ for Fisher's $\uparrow^{\ddagger}$ and GTest $^{*}$

Branch no. RI RV SI SV G-value

rhodopsin

$\begin{array}{llllll}0 & 0 & 2 & 0 & 0 & 0.00 \\ 1 & 0 & 2 & 0 & 0 & 0.00 \\ 2 & 0 & 2 & 0 & 0 & 0.00 \\ 3 & 0 & 5 & 0 & 0 & 0.00 \\ 4 & 0 & 5 & 0 & 0 & 0.00 \\ 5 & 0 & 5 & 0 & 0 & 0.00 \\ 6 & 0 & 5 & 0 & 0 & 0.00 \\ 7 & 0 & 5 & 0 & 0 & 0.00 \\ 8 & 0 & 5 & 0 & 0 & 0.00 \\ 9 & 0 & 5 & 0 & 0 & 0.00 \\ 10 & 0 & 5 & 0 & 0 & 0.00 \\ 11 & 0 & 8 & 0 & 0 & 0.00 \\ 12 & 0 & 8 & 0 & 0 & 0.00 \\ 13 & 0 & 8 & 0 & 0 & 0.00 \\ 14 & 0 & 8 & 0 & 0 & 0.00 \\ 15 & 0 & 8 & 0 & 0 & 0.00 \\ 16 & 0 & 11 & 1 & 0 & 2.01 \\ 17 & 0 & 0 & 1 & 0 & 0.00 \\ 18 & 0 & 9 & 0 & 0 & 0.00 \\ 19 & 0 & 11 & 0 & 0 & 0.00 \\ 21 & 12 & 1 & 0 & 2.2 \\ 24 & 0 & 12 & 0 & 0 & 0.00 \\ 23 & 0 & 0 & 0 & 2.2 \\ 21 & 0 & 0 & 2.2\end{array}$

PeerJ reviewing PDF | (v2014:01:1344:2:1:NEW 24 Jul 2014) 
25

$\begin{array}{lllll}0 & 12 & 1 & 0 & 2.2\end{array}$

26

$\begin{array}{lllll}1 & 12 & 2 & 0 & 5.5^{\dagger}\end{array}$

27

$\begin{array}{lllll}8 & 24 & 5 & 1 & 6.76^{*}\end{array}$

